\title{
Microbial Communities Associated with Potato Common Scab-Suppressive Soil Determined by Pyrosequencing Analyses
}

Noah Rosenzweig, Department of Plant Pathology, James M. Tiedje, Department of Crop and Soil Science and Center for Microbial Ecology, John F. Quensen, III, Department of Crop and Soil Science and Center for Microbial Ecology, Qingxiao Meng, Department of Plant Pathology, and Jianjun J. Hao, Department of Plant Pathology, Michigan State University, East Lansing 48824

\begin{abstract}
Rosenzweig, N., Tiedje, J. M., Quensen, J. F., III, Meng, Q., and Hao, J. J. 2012. Microbial communities associated with potato common scabsuppressive soil determined by pyrosequencing analyses. Plant Dis. 96:718-725.

Potato common scab, caused by Streptomyces spp., is an annual production problem for potato growers, and not effectively controlled by current methods. A field with naturally occurring common scab suppression has been identified in Michigan, and confirmed to have a biological basis for this disease suppression. This field and an adjacent scab nursery conducive to disease were studied using pyrosequencing to compare the two microbial communities. Total DNA was extracted from both the disease-conducive and -suppressive soils. A phylogenetically taxon-informative region of the 16S rRNA gene was used to establish operational taxonomic units (OTUs) to characterize bacterial

community richness and diversity. In total, 1,124 OTUs were detected and 565 OTUs (10\% dissimilarity) were identified in disease-conducive soil and 859 in disease-suppressive soil, including 300 shared both between sites. Common phyla based on relative sequence abundance were Acidobacteria, Proteobacteria, and Firmicutes. Sequences of Lysobacter were found in significantly higher numbers in the diseasesuppressive soil, as were sequences of group 4 and group 6 Acidobacteria. The relative abundance of sequences identified as the genus Bacillus was significantly higher by an order of magnitude in the disease-conducive soil.
\end{abstract}

Potato common scab (PCS), caused by Streptomyces spp., is an annual production problem for potato growers worldwide $(26,43)$. The disease is of particular importance in Michigan and the United States North-Central region, where disease development can be severe due to favorable environmental conditions, including warm, dry seasons with soil temperatures above $22^{\circ} \mathrm{C}$ (44). PCS pressure can vary between years, locations, and potato varieties (52). Consistent and effective management of PCS has remained elusive $(26,43)$. Few commercially important potato varieties show significant resistance to PCS under variable production conditions (54). PCS is of particular concern in chipping varieties where the disease impact is economic due to cosmetic symptoms on the tuber surface. Cultural practices, including planting certified disease-free tubers, rotating crops, maintaining soil moisture during tuber formation, lowering soil $\mathrm{pH}$, and chemically fumigating soils, provide inconsistent results or fail to significantly reduce pathogen inoculum levels (14). Most cultural practices for disease control are related to enhancing soil microbial activities (18). Because modification of microbial communities by these methods is indirect and the mechanisms involved not well known, inconsistency in disease control is understandable. To overcome this limitation, studies of naturally occurring disease-suppressive soils may provide a baseline for characterization of important microbial populations that contribute to disease suppressiveness, and aid the development of a sustainable alternative to current control strategies.

\section{Corresponding author: J. Hao, E-mail: jjhao@msu.edu}

Data deposition: bacterial 16S rDNA sequences reported in this paper have been deposited in the EMBL European Read Archive (accession number ERP000800).

* The $e$-Xtra logo stands for "electronic extra" and indicates that two supplemental tables are included in the online edition.

Accepted for publication 27 November 2011.

http://dx.doi.org/10.1094/PDIS-07-11-0571

(C) 2012 The American Phytopathological Society
With recent advances in culture-independent approaches to microbial community analysis, there has been renewed interest in the phenomena of endemic suppression of soilborne plant diseases, including exploration of the effects of plant species and soil management on disease suppression and the associated microbial community structure. Intrinsic disease suppression in agricultural soils has long been known and has been explored in multiple pathogenplant systems $(2,8,33,50)$. PCS disease suppression is found in cultivated soils $(4,22)$ and appears to be related to changes in microbial populations. Disease control is largely attributed to biological interactions (e.g., parasitism and competition) between antagonistic microflora and pathogens mediated via antibiotic production or enzymatic activity (3). Characterization of the native potato-associated soil microbial community in disease-suppressive soils offers new opportunities for managing native soil microbes to control PCS.

The development of newer sequencing technologies means that soil microbial communities, including those of PCS diseasesuppressive soils, can be studied in greater detail than was previously possible with earlier, lower-resolution culture-independent techniques. For example, identification of microorganism on selective or semiselective media can only recover less than $5 \%$ of microorganisms in the whole community (47), and a priori community nutrient-use profiling (BIOLOG plate; Hayward, CA) and fatty acid methyl ester methods are highly dependent on sample preparation and current available databases $(38,40)$. Terminal restriction fragment length polymorphism (T-RFLP) and denaturing gradient gel electrophoresis cannot give precise species and taxonomic identity $(27,32)$. The recent accessibility of the pyrosequencing platform has also enabled relatively affordable analysis of microbial community structure of the soil $(16,23)$. Pyrosequencing as implemented in the Roche Genome Sequencer FLX Titanium is a high-throughput next-generation platform capable of producing approximately $10^{6}$ sequence reads of $400 \mathrm{bp}$ in a single run, and provides greater depth than traditional DNA sequencing technologies and, hence, detection of rare species (16).

We identified a field in East Lansing, MI that exhibited natural suppression to PCS after consecutive years of potato production (32). In culture-dependent studies, dilution plating showed no clear trends in differences in populations of total fungi, total bacteria, 
streptomycetes, fluorescent pseudomonads, and bacilli, but TRFLP analysis revealed distinct microbial community structure of suppressive and conducive soils (32). With this concurrent study, it was possible to link genus-level taxonomic identity and compare microbial community structure of the suppressive soil with an adjacent nonsuppressive disease-infected field, using a pyrosequencing approach. Disease suppression is largely attributed to biological interactions (e.g., parasitism and competition) between antagonistic microflora and pathogens mediated via antibiotic production or enzymatic activity $(4,22,32)$. We hypothesize that this disease-suppressive soil has a characteristic microbial community structure. We amplified the variable region V4-V5 because it is more conserved in length which, within the highly conserved 16S rRNA gene, aids in quality control and reduced computer processing time without a loss of taxonomic resolution of soil microbial communities.

\section{Materials and Methods}

The culture-independent approach consisted of (i) pyrosequencing of ribosomal 16S V4-V5 fragment amplicons using DNA barcoding; (ii) computational sorting of resulting bar-coded sequences into conducive soil (CS) and suppressive soil (SS) sources; (iii) clustering the resulting sequences into operational taxonomic units (OTUs); (iv) comparison of sequences using phylotype analyses based on the Ribosomal Database Project (RDP) taxonomy; and (v) statistical analysis of frequency distributions; OTU richness, diversity, and relative abundance of predominant taxa in CS and SS.

Soil sampling and DNA extraction. Soil samples were collected during tuber bulking from the root-soil interface of potato plants ('Snowden') from CS and SS in two adjacent fields (CS: $42^{\circ} 42^{\prime} 56.35^{\prime \prime} \mathrm{N}, \quad 84^{\circ} 27^{\prime} 56.35^{\prime \prime} \mathrm{W}$, elevation $262 \mathrm{~m}$ and SS: $42^{\circ} 43^{\prime} 01.31^{\prime \prime} \mathrm{N}, 84^{\circ} 27^{\prime} 57.11^{\prime \prime} \mathrm{W}$, elevation $262 \mathrm{~m}$ ) with a similar history of continuous potato monoculture, in East Lansing, MI. Each field measured 30 by $60 \mathrm{~m}$. A composite of three soil cores within $1 \mathrm{~m}^{2}$ from five random soil sampling sites were collected for each CS and SS field ( $n=10$ soil sampling sites total). Individual sampling sites were at least $3 \mathrm{~m}$ apart. At each sampling site, soil cores were collected with a $25-\mathrm{mm}$ JMC soil corer (Clements Assoc., Newton, IA) to a depth of $100 \mathrm{~mm}$. The soil corer was disinfested with $10 \% \mathrm{NaClO}$ followed by $70 \% \mathrm{EtOH}$ and the composite soil cores (approximately $300 \mathrm{~g}$ ) placed into separate plastic bags, transported to the laboratory on ice, and stored at $4^{\circ} \mathrm{C}$ pending further analysis. Subsequently, total genomic DNA was extracted from a half-gram of soil from each composite soil sample using the FastDNA SPIN Kit for Soil (MP Biomedicals LLC, Solon, $\mathrm{OH}$ ) according to the manufacturer's instructions. DNA samples were stored at $-20^{\circ} \mathrm{C}$ until analysis.

Polymerase chain reaction amplification of soil sample DNA and sample pooling for pyrosequencing. DNA extracted from each soil sample served as a template in triplicate polymerase chain reactions (PCRs; 30 reactions total) performed using the Roche High-Fidelity PCR system (Roche Diagnostics GmbH, Mannheim, Germany). Each 20- $\mu$ l reaction contained $1.8 \mathrm{mM}$ $\mathrm{MgCl}_{2}, 400 \mathrm{nM}$ each primer, $200 \mu \mathrm{M}$ each dNTP (Invitrogen Life Technologies, Carlsbad, CA), $4 \mu \mathrm{g}$ of bovine serum albumin (New England Biolabs, Ipswich, MA), $1 \mathrm{U}$ of Taq polymerase, and $50 \mathrm{ng}$ of template DNA. The forward primer consisted of the 25-bp $454 \mathrm{~A}$ Adapter and a 10-bp barcode, followed by the 15-bp universal bacterial primer $16 \mathrm{~S}-577 \mathrm{~F}$ (5'-CGTATCGCCTCCCTCGCGCCA TCAGbarcodeAYTGGGYDTAAAGNG-3'). The reverse primer consisted of the 25-bp 454 B Adapter and the 18-bp universal bacterial primer 16S-926R (5'-CTATGCGCCTTGCCAGCCCGC TCAGCCGTCAATTCMTTTRAGT-3'). These primers target an approximately 331-bp region of the 16S rRNA gene containing variable regions 4 and 5 . This region was chosen because it is phylogenetically informative (variable across taxa) and is a length well suited for pyrosequencing (51). Following pyrosequencing, the 10bp barcode enabled the sorting of sequences by original soil sampling site. Amplifications were performed on an Eppendorf Mastercycler thermocycler (Eppendorf North America, Hauppauge, NY) using the following temperature program: $3 \mathrm{~min}$ at $94^{\circ} \mathrm{C}$; 30 cycles of $45 \mathrm{~s}$ at $94^{\circ} \mathrm{C}, 45 \mathrm{~s}$ at $56^{\circ} \mathrm{C}$, and 1 min at $72^{\circ} \mathrm{C}$; followed by final extension for $7 \mathrm{~min}$ at $72^{\circ} \mathrm{C}$.

PCR amplicon libraries were purified on a $1.2 \%$ agarose gel. DNA was extracted from the gel slices using a QIAquick gel extraction kit (Qiagen, Maryland) per the manufacturer's directions. DNA was eluted from the filter with $30 \mu \mathrm{l}$ of elution buffer and purified a second time using a Qiagen PCR purification kit. Each purified reaction sample was quantified using the PicoGreen dsDNA Assay Kit (Invitrogen Life Technologies) and the Qubit fluorometer (Invitrogen Life Technologies) according to the manufacturer's instructions. Equal DNA masses of 10 separate samples representing all 10 soil cores from each field were combined, the total concentration adjusted to $2 \mathrm{ng} / \mu \mathrm{l}$, and the pooled sample was sent to the Research Technology Support Facility at Michigan State University (East Lansing) for emulsion PCR, GS amplicon library preparation, and pyrosequencing on a 454 Life Sciences GS-FLX instrument (Roche).

Bioinformatics and computational and statistical analyses. Bioinformatics and computational analyses were performed remotely in the Computational Genetics Laboratory at the Minnesota Supercomputing Institute for Advanced Computational Research at the University of Minnesota Microbial and Plant Genomics Institute. All statistical analyses were performed using the R-statistical package (v.2.10; http://cran.r-project.org/) or SAS v. 9.2 TS Level. Generated sequence data have been deposited in European Molecular Biology Laboratory European Read Archive using the RDP Short Read Archive Prepkit (http://pyro.cme.msu.edu/).

Operational taxonomic unit analysis of richness and diversity: rarefaction, Chao1, ACE, Shannon, and Simpson indices. OTU analyses were performed using the MOTHUR software package (v.1.17.0) (39). OTU calculations were based on the genetic distance between sequences and used to estimate the richness and diversity of a sampled community. For alignment purposes only, quality-filtered unique sequences (minimum length $>150 \mathrm{bp}$ ) were included. Chimeric sequences were removed and a preclustering step was used to reduce noise to signal from raw pyrosequencing data prior to OTU analysis. Sequences were then aligned to the SILVA alignment database (http://www.arb-silva.de/) using the Needleman-Wunsch pairwise alignment method with a kmer size of 8 and a reward of +1 for a match and penalties of -1 and -2 for a mismatch and gap, respectively. Subsequently, vertical gaps were removed to minimize the alignment sequence. The reference database was used to produce an alignment to cover the entire $16 \mathrm{~S}$ rRNA gene. A PHYLIP-formatted distance matrix was calculated for distances equal to and smaller than 0.10 . Sequences were clustered and assigned to OTUs at distances between 0.0 and 0.10 in 0.01 increments. The clusters served as the OTUs for generating predictive rarefaction models and for calculating ACE (6) and Chaol (7) richness indices. The number of OTUs and the ACE and Chao1 richness estimates for the unique, 0.03, 0.05, and 0.10 OTU definitions were calculated. Single and multiple intrasample rarefaction curves were generated using a resampling-without-replacement approach. The Shannon (41) and Simpson (42) diversity indices of each sample for unique, 0.03, 0.05, and 0.10 OTU definitions were calculated.

Phylotype and statistical analyses. Bioinformatics and computational biology approaches were used to assign taxonomy information to compare the associated soil microbial communities' composition of CS and SS. A Bayesian approach was used to assign taxonomic identity to sequences. The phylotype of individual sequence OTUs was determined using the RDP6 taxonomy at various levels of taxonomic hierarchy. Finally, the resulting matches for each set of sequence data were summarized at various levels of taxonomic hierarchal structure (e.g., phylum and class). Kolmogorov-Smirnov, analysis of variance, and Wilcoxon nonparametric analyses were performed on taxonomy results.

\section{Results}

Bacterial communities associated with CS and SS. Richness estimates of bacteria associated with CS and SS. In total, 66,308 
high-quality sequences were obtained from the 10 individual soil DNA samples (Table 1). To discriminate OTUs, rarefaction curves were calculated on the complete data set using a range of dissimilarity values (unique, 3, 5 and 10\%; Fig. 1). Community diversity and richness are reported for $10 \%$ similarity (Table 2 ). Subsequent community comparison ( $\beta$-diversity) analyses focused on defining OTUs at the conservative $10 \%$ dissimilarity, because the rarefaction curves suggest the greatest potential for comprehensive sampling of the soil communities at this dissimilarity level.

Among all soil samples, there were 1,124 distinct OTUs observed at $10 \%$ dissimilarity (Table 2; Fig. 2). In all, $46 \%$ (521) of all OTUs were represented by a single sequence (singletons), and $27 \%$ (300) of OTUs were observed in both CS and SS. CS samples had a total of 565 OTUs and SS samples supported 859 OTUs in 21,110 and 23,342 sequences, respectively (Table 2; Fig. 2). On average, individual SS samples supported a greater number of OTUs than CS samples; average OTU numbers per soil type were 318 and 471 for CS and SS, respectively. The total number of OTUs represented once (singletons) + OTUs represented twice (doubletons) associated with soil type was 314 (55.6\% of all OTUs on that soil type) and 531 (61.8\% of all OTUs on that soil type) for CS and SS, respectively (data not shown).

Bacterial community richness and diversity among soil types. Mean bacterial OTU richness (number of OTUs per soil sample) and diversity were consistently higher for SS than for CS, as were both the mean ACE and Chao1 estimates of OTU richness at 3, 5 and 10\% dissimilarity (Table 2; Supplementary Tables S1 and S2). Moreover, the mean Shannon and Simpson diversity indices for individual plants were higher for SS than CS at 3,5 and 10\% dissimilarity (Table 2).

Phylotype-based analysis of bacterial community composition. DNA sequence reads were compared against the RDP6 taxonomy classification to assign best-match taxonomic sequence identity using an $80 \%$ cutoff confidence interval. In total, 20 bacterial phyla, 48 bacterial classes, 86 bacterial orders, 172 bacterial families, and 334 bacterial genera were identified based on percent sequence similarity (Table 1).

Relative abundance of bacterial taxa. DNA sequence assignment was dominated by 5 of the 20 bacterial phyla recovered, comprising nearly $87 \%$ of the total bacterial community assemblage (Table
$3)$. These phyla were present in every soil sample and consisted of major bacterial groups, including Proteobacteria (39\%), Acidobacteria (14\%), Gemmatimonadetes (12\%), and Actinobacteria (7\%), with unclassified bacteria representing $13 \%$ of the total bacterial phyla. Bacterial phyla that were unique to a field represented less than $1 \%$ of the total and were typically represented as singletons from only one sample (data not shown). There were significant differences in relative abundance (based on mean number of sequence reads) between the soil conditions for five bacterial phyla ( $\alpha=0.05$, Wilcoxon; Table 3$)$. CS had a significantly greater abundance of the phyla Deinococcus-Thermus (CS: $5.6 \%$; SS: 2.6\%) and Firmicutes (CS: 7.3\%; SS: 4.5\%). Although SS had a greater abundance of the phyla BRC1 and WS3, these phyla, in

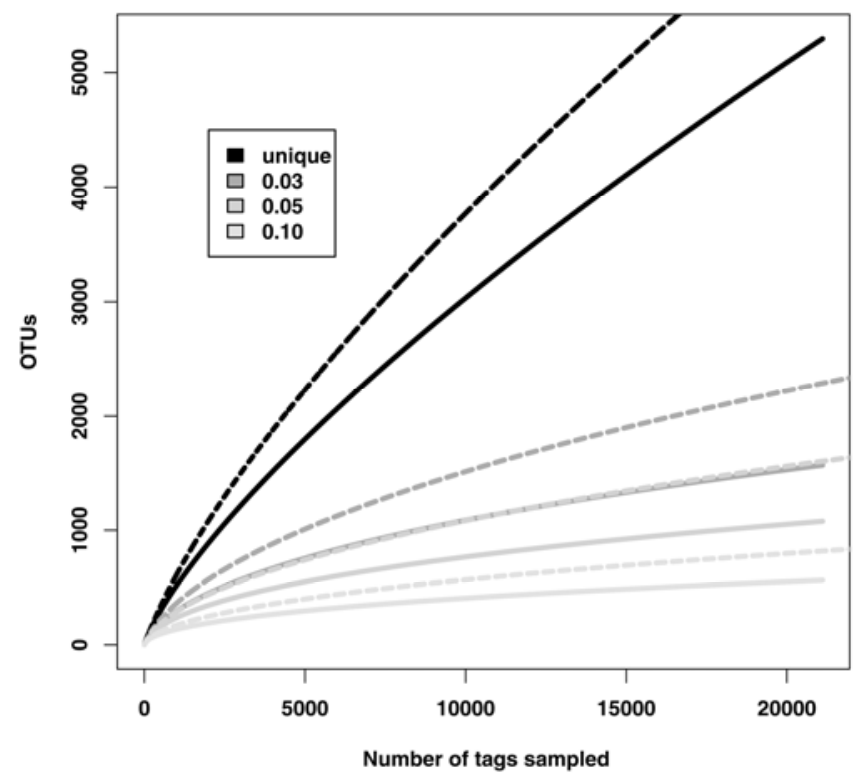

Fig. 1. Rarefaction analysis curves generated for operational taxonomic units (OTUs) that contain unique sequences and for sequence dissimilarities that do not exceed 3,5 , or $10 \%$. Solid lines represent samples from disease-conducive soils (CS), and dashed lines represent samples from disease-suppressive soils (SS).

Table 1. Total number of sequences, average number of sequences per plant, and total bacterial phyla, families, and genera classified from disease-conducive and -suppressive soil (CS and SS, respectively)

\begin{tabular}{|c|c|c|c|c|c|c|c|}
\hline \multirow[b]{2}{*}{ Number of samples (soil) } & \multirow[b]{2}{*}{ Total number of sequences } & \multirow[b]{2}{*}{ Sequences/sample (range) ${ }^{\mathbf{b}}$} & \multicolumn{5}{|c|}{ Classification $^{a}$} \\
\hline & & & Phyla & Classes & Orders & Families & Genera \\
\hline $5(\mathrm{CS})$ & 32,174 & $6,438(5,851-7,417)$ & 19 & 46 & 66 & 142 & 256 \\
\hline $5(\mathrm{SS})$ & 34,134 & $6,827(6,154-7,246)$ & 18 & 42 & 83 & 163 & 298 \\
\hline 10 (Total) & 66,308 & $6,631(5,851-7,417)$ & 20 & 48 & 86 & 172 & 334 \\
\hline
\end{tabular}

${ }^{a}$ Taxonomic classification level was determined based on the RDP6 taxonomy outline.

${ }^{\mathrm{b}}$ Average number of sequences/soil sample (range).

Table 2. Richness and diversity of operational taxonomic units (OTUs), which were determined based upon $10 \%$ dissimilarity of disease-conducive and -suppressive soil (CS and SS, respectively)

\begin{tabular}{lrccccc}
\hline Sample $^{\mathbf{a}}$ & Reads & OTU & ACE (range) & Chao1 (range) & Shannon (range) & Simpson (range) \\
\hline CS1 & 3,376 & 286 & $535(477,610)$ & $424(372,510)$ & $4.08(4.02,4.14)$ & $0.051(0.046,0.055)$ \\
CS2 & 3,960 & 206 & $465(404,545)$ & $396(314,541)$ & $2.69(2.63,2.76)$ & $0.201(0.191,0.210)$ \\
CS3 & 4,878 & 442 & $970(877,1084)$ & $736(642,873)$ & $4.67(4.63,4.72)$ & $0.024(0.022,0.025)$ \\
CS4 & 3,978 & 334 & $833(743,944)$ & $558(479,678)$ & $4.15(4.09,4.20)$ & $0.046(0.043,0.050)$ \\
CS5 & 3,745 & 322 & $686(612,778)$ & $560(473,698)$ & $4.25(4.20,4.30)$ & $0.037(0.034,0.040)$ \\
CS total & 21,110 & 565 & $1,195(1094,1314)$ & $992(865,1172)$ & $3.82(3.79,3.84)$ & $0.058(0.056,0.060)$ \\
SS1 & 4,910 & 502 & $1,177(1070,1304)$ & $969(829,1169)$ & $4.04(3.98,4.11)$ & $0.106(0.098,0.114)$ \\
SS2 & 4,691 & 193 & $471(406,557)$ & $352(285,471)$ & $1.48(1.41,1.55)$ & $0.608(0.590,0.626)$ \\
SS3 & 4,525 & 526 & $1,341(1218,1485)$ & $894(788,1042)$ & $4.90(4.85,4.94)$ & $0.017(0.016,0.018)$ \\
SS4 & 4,487 & 513 & $1,260(1145,1396)$ & $982(843,1179)$ & $4.46(4.40,4.52)$ & $0.056(0.051,0.061)$ \\
SS5 & 4,672 & 621 & $1,542(1411,1694)$ & $1,152(1009,1348)$ & $5.09(5.05,5.14)$ & $0.014(0.014,0.015)$ \\
SS total & 23,342 & 859 & $2,073(1,923,2,244)$ & $1,512(1357,1714)$ & $3.81(3.78,3.84)$ & $0.097(0.094,0.100)$ \\
\hline
\end{tabular}

${ }^{\text {a }}$ CS 1 to $-5=$ CS samples 1 to 5 and SS 1 to $-5=$ SS samples 1 to 5 . 
both cases and taken together, represented less than $1 \%$ of the relative abundance of the total phyla identified (Table 3 ).

The top 10 of the 48 classes, 86 orders, and 172 families recovered comprised nearly 85,75 , and $70 \%$, respectively, of the total bacterial community assemblage (data not shown). Moreover, 24 families of bacteria differed significantly in mean relative abundance between CS and SS samples (Wilcoxon, $\alpha=0.05$; Table 4). The most abundant families were from some phylogenetically well-established representative soil groups such as Bradyrhizobiaceae $(5.4 \%)$, Gemmatimonadaceae (13\%), Nocardioidaceae $(2.3 \%)$, Planctomycetaceae $(1.8 \%)$, Trueperaceae (3.5\%), Xanthomonadaceae (23\%), an unclassified Acidobacteria group 11 (4.2\%), an unclassified Acidobacteria group $3(2.5 \%)$, an unclassified Acidobacteria group 6 (12\%), and an unclassified WS3 (13\%) (data not shown). Moreover 21 families of bacteria differed significantly in mean relative abundance between CS and SS samples (Wilcoxon, $\alpha=0.05$; Table 4). Overall, CS samples had a greater mean abundance of Acetobacteraceae (CS: $1.2 \%$; SS: $0.5 \%$ ) and Bacillaceae (CS: 1.8\%; SS: 0.2\%), while SS samples had a greater mean abundance of unclassified Acidobacteria group 6 (CS: $1.2 \%$; SS: $8.6 \%$ ), Nocardioidaceae (CS: $1.0 \%$; SS: 2.6\%), Pseudomonadaceae (CS: $0.04 \%$; SS: $0.5 \%$ ), unclassified Acidobacteria group 11 (CS: 0.0\%; SS: 0.2\%), and unclassified Bacilli (CS: $0.04 \%$; SS: $0.4 \%$ ) (Table 4). The remaining classes that were significantly different had a combined mean relative abundance of less than $1 \%$ for both CS and SS samples (Table 4).

Abundance distribution and composition of bacterial communities. The sequence abundance distributions for bacterial genera and species were compared among samples. Combining data from all samples, there was a significant difference (Kolmogorov-Smirnov test, $P=0.01)$ in the distribution of sequences among bacterial genera between soil types. In total, 10 of the 334 bacterial genera recovered comprised nearly $65 \%$ of the total bacterial community assemblage. These included taxa from some common soil groups such as Bradyrhizobium (2.4\%), Gemmatimonas (12.6\%), Rhodanobacter (19.3\%), Truepera (2.6\%), unclassified Xanthomonadaceae $(2.7 \%)$, unclassified Acidobacteria group 1 (3.5\%), unclassified Acidobacteria group $3(2.3 \%)$, unclassified Acidobacteria group 6 (5.4\%), unclassified Hyphomicrobiaceae 77 $(1.5 \%)$, and unclassified $129(12.6 \%)$ that was unrelated to any known bacterial lineage (data not shown). Moreover, 41 genera of bacteria differed significantly in mean relative abundance between CS and SS samples ( $\alpha=0.05$, Wilcoxon; Table 5). Overall, CS samples had a greater mean abundance of Bacillus (CS: 1.4\%; SS:
0.1\%), Trupera (CS: 5.5\%; SS: 0.07\%), unclassified Bacillales (CS: $1.3 \%$; SS: $0.3 \%$ ), and unclassified Bradyrhizobiaceae (CS: $2.6 \%$; SS: $0.5 \%$ ), while SS samples had a greater mean abundance of Lysobacter (CS: 0.04\%; SS: 0.2\%), Rhizobium (CS: 0.08\%; SS: $0.2 \%$ ), unclassified group 4 (CS: $0.2 \%$; SS: $2.3 \%$ ), and unclassified group 6 (CS: 1.3\%; SS: 9.0\%) (Table 5).

\section{Discussion}

Different soil types such as bulk and rhizosphere soils can support distinct microbial communities $(12,19,24,28,45)$. We have found a diverse assemblage of microbial populations in the soil microbial communities associated with disease-conducive and -suppressive soils, which is supported by other work $(20,57,59)$. There were significant differences in some of the most abundant taxa identified between CS and SS. CS and SS types were also distinguished from one another by the difference in relative abundance of key bacterial taxa, indicating differential community structure that may be linked to the soil disease profile and provide proxies indicating disease suppression.

The biological factors contributing to the development of disease-suppressive soil may vary due to the complexity of microbemicrobe interactions $(1,15,33,49)$ and the diversity of microbes inhabiting the soil $(47,48)$. For example, naturally occurring PCS-

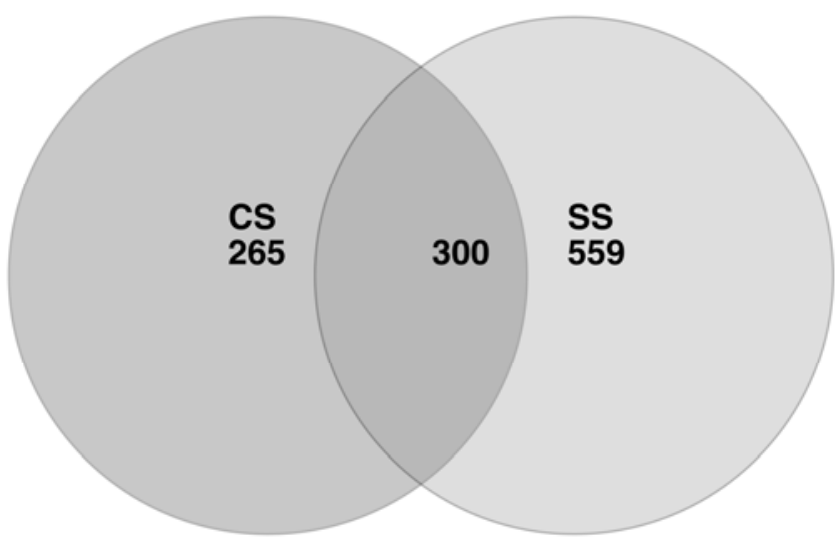

Fig. 2. Venn diagram indicating shared and unique observed operational taxonomic units (OTUs) at $10 \%$ dissimilarity between disease-conducive-soil (CS) and disease-suppressive soil (SS). Numbers of OTUs in groups CS, SS, and shared between groups are shown.

Table 3. Relative sequence abundance of recovered soil bacterial classes from soil samples divided into categories of soil disease profile and combined totals

\begin{tabular}{|c|c|c|c|}
\hline \multirow[b]{2}{*}{ Phylumb } & \multicolumn{3}{|c|}{ Mean relative abundance $(\mathrm{SE})^{\mathrm{a}}$} \\
\hline & CS & SS & Total \\
\hline Proteobacteria & $34.693( \pm 15.515)$ & $43.191( \pm 19.316)$ & $38.942( \pm 17.415)$ \\
\hline Unclassified & $13.394( \pm 5.990)$ & $12.716( \pm 5.687)$ & $13.055( \pm 5.838)$ \\
\hline Gemmatimonadetes & $12.536( \pm 5.606)$ & $12.231( \pm 5.470)$ & $12.383( \pm 5.538)$ \\
\hline Acidobacteria & $12.019( \pm 5.375)$ & $16.483( \pm 7.371)$ & $14.251( \pm 6.373)$ \\
\hline Actinobacteria & $7.690( \pm 3.439)$ & $7.013( \pm 3.136)$ & $7.352( \pm 3.288)$ \\
\hline Firmicutes* & $7.339( \pm 3.282)$ & $2.027( \pm 0.907)$ & $4.683( \pm 2.094)$ \\
\hline Deinococcus-Thermus* & $5.628( \pm 2.517)$ & $0.090( \pm 0.040)$ & $2.859( \pm 1.279)$ \\
\hline Chloroflexi & $1.986( \pm 0.888)$ & $2.731( \pm 1.222)$ & $2.359( \pm 1.055)$ \\
\hline Planctomycetes & $1.826( \pm 0.817)$ & $1.074( \pm 0.480)$ & $1.450( \pm 0.648)$ \\
\hline Cyanobacteria & $1.570( \pm 0.702)$ & $0.073( \pm 0.033)$ & $0.822( \pm 0.367)$ \\
\hline Bacteroidetes & $0.597( \pm 0.267)$ & $0.288( \pm 0.129)$ & $0.442( \pm 0.198)$ \\
\hline Verrucomicrobia & $0.572( \pm 0.256)$ & $1.323( \pm 0.592)$ & $0.947( \pm 0.424)$ \\
\hline Nitrospira & $0.060( \pm 0.027)$ & $0.112( \pm 0.050)$ & $0.086( \pm 0.038)$ \\
\hline WS $3 *$ & $0.040( \pm 0.018)$ & $0.464( \pm 0.207)$ & $0.252( \pm 0.113)$ \\
\hline OP10 & $0.025( \pm 0.011)$ & $0.073( \pm 0.033)$ & $0.049( \pm 0.022)$ \\
\hline Unclassified bacteria* & $0.025( \pm 0.011)$ & $0.060( \pm 0.027)$ & $0.043( \pm 0.019)$ \\
\hline OD1 & $0.005( \pm 0.002)$ & $\ldots$ & $0.003( \pm 0.001)$ \\
\hline Spirochaetes & $0.005( \pm 0.002)$ & $\ldots$ & $0.003( \pm 0.001)$ \\
\hline $\mathrm{BRC} 1 *$ & $\ldots$ & $0.052( \pm 0.023)$ & $0.026( \pm 0.012)$ \\
\hline
\end{tabular}

a Mean relative abundance based on percentage of recovered sequences; SE $=$ standard error of the mean.

b Taxonomic classification level was determined based on the RDP6 taxonomy outline; * indicates a significant difference between means of diseaseconducive and -suppressive soil (CS and SS, respectively) based on Wilcoxon analysis; comparisons were statistically different at $\alpha=0.05$. 
suppressive soils are generally found following long-term potato monoculture $(25,33)$ and, in several cases, nonpathogenic Streptomyces spp. are a key factor for disease suppression $(4,32,40,53)$. However, other microorganisms may also be important $(4,31$, 40,46).

The combined use of a curated database alignment (e.g., SILVA) and the RDP6 taxonomy enhanced the number of OTUs identified from recovered sequences while providing the added taxonomic identification of the sequences. Many sequences still remain unclassified due to database reliance on well-sampled organisms whose genomes are annotated. Moreover, based on estimates calculated by rarefaction curves (Fig. 1), it is likely that our results represent only a fraction of the wealth of bacterial diversity that exists in soil microbial communities in cultivated soils. Although an average of 6,631 sequences per soil sample were recovered, the entirety of bacterial richness at the species level within an individual sample was not achieved. Therefore, the large size (CFU per gram of soil) and high diversity and complexity of soil communities $(11,48)$ will require future effort in more extensive sequencing to comprehensively characterize community composition, and to ultimately attribute function to distinct bacterial taxa responsible for disease suppression. In spite of this limited characterization of the microbial community, the taxa that dominate in abundance may be key in maintaining disease levels in SS systems.

Pyrosequencing was used successfully to detect a multitude of bacteria inhabiting cultivated soil, including a large proportion of unclassified taxa that are presumably unculturable to date. This is complementary to and confirmation of our results using culturebased methods (32). The two methods together reveal the soil microbial community associated with disease-suppressive soil. Pyrosequencing technology brings the advantage of depth of detection compared with the culture- and non-culture-based studies that have typically focused on specific taxa or functional groups $(22,29,30,35,37,40,45,55,56)$. This study found major bacterial taxa that are common to most soil types at high levels of relative abundance similar to previous work $(57,58)$ : class $\alpha$-Proteobacteria and phyla Actinobacteria, Acidobacteria, Verrucomicrobia, Proteobacteria, and Firmicutes. Conversely, two phyla that were poorly represented by low relative abundance were the recently recognized OD1 and the Spirochaetes. As the DNA database develops, we anticipate that the unclassified DNA reads corresponding to different taxa, as shown with previous studies (5), will be identified.

According to results from the OUT-based analyses, $73 \%$ of the taxa were unique to CS and SS. This agrees with results from TRFLP 16S rDNA analyses, in which distinct microbial communities were distinguished by SS and CS (32). Moreover these results are both quantitative and qualitative by identifying the taxa unique to CS and SS and, more importantly, their relative abundance, whereas T-RFLP is able to distinguish communities but not their respective constituents.

The Proteobacteria was the most abundant phylum identified. This phyla has been found previously in Brazilian Atlantic Forest soils (10) and agricultural soils (57). Within the $\gamma$-Proteobacteria, the genus Lysobacter belonging to the family Xanthomonadaceae was in significantly higher numbers in SS. Similar results have been found in soils suppressive to Rhizoctonia solani AG2.2IIIB in sugar beet and Streptomyces scabies in radish correlated positively with the presence of antagonistic Lysobacter spp. and Streptomyces spp., respectively (34). Moreover, these results are consistent with previous work on disease-suppressive bacteria that identified $\gamma$ - and $\beta$-Proteobacteria (Pseudomonadaceae, Burkholderiaceae, and Xanthomonadales) and the Firmicutes (Lactobacillaceae) as the most dynamic taxa associated with disease suppression (31). Therefore, continued species-level and functional analyses of the genus Lysobacter may identify members of this group effective in biologically based plant disease management.

The Acidobacteria was the second most abundant phyla identified in this study, which is found in diverse soil types from North and South America $(17,20)$, active in cultivated wheat (57) associated with later stages of the take-all decline disease (36), and in the rhizosphere of chestnut trees (21). Group 4 and 6 Acidobacteria were in significantly higher numbers in SS samples. These groups are linked to $\mathrm{pH}$ ranges $(17,20,57)$, which are within the range of the $\mathrm{pH}$ of the soil in this study (32). Moreover, Acidobacteria group 4 is enhanced in wheat-soybean rotation management practices (57). Acidobacteria groups sensitive to some cultural practices may provide important indicators of disease suppression.

Members of the phyla Actinobacteria, commonly found among multiple soil samples in previous studies $(20,57)$ and in SS (31), were among the most abundant recovered from both CS and SS

Table 4. Relative sequence abundance of significantly different bacteria from soil samples divided into categories of soil disease profile and combined totals

\begin{tabular}{|c|c|c|c|}
\hline \multirow[b]{2}{*}{ Family $^{\mathbf{b}}$} & \multicolumn{3}{|c|}{ Mean relative abundance $(\mathrm{SE})^{\mathrm{a}}$} \\
\hline & $\mathrm{CS}^{\mathbf{c}}$ & $\mathbf{S S}^{\mathbf{c}}$ & Total \\
\hline Acetobacteraceae & $1.254( \pm 0.124)$ & $0.490( \pm 0.099)$ & $0.872( \pm 0.677)$ \\
\hline Actinospicaceae & $0.206( \pm 0.096)$ & $0.009( \pm 0.005)$ & $0.107( \pm 0.105)$ \\
\hline Bacillaceae & $1.756( \pm 0.846)$ & $0.198( \pm 0.028)$ & $0.977( \pm 0.892)$ \\
\hline Clostridiaceae & $1.079( \pm 0.249)$ & $0.391( \pm 0.084)$ & $0.735( \pm 0.581)$ \\
\hline Comamonadaceae & $0.030( \pm 0.020)$ & $0.240( \pm 0.067)$ & $0.135( \pm 0.049)$ \\
\hline Deinococcaceae & $0.110( \pm 0.055)$ & $0.009( \pm 0.005)$ & $0.059( \pm 0.058)$ \\
\hline Ilumatobacter & $\ldots$ & $0.086( \pm 0.018)$ & $0.043( \pm 0.009)$ \\
\hline Incertae Sedis XVIII & $0.030( \pm 0.018)$ & $0.150( \pm 0.030)$ & $0.090( \pm 0.030)$ \\
\hline Mycobacteriaceae & $0.291( \pm 0.049)$ & $0.116( \pm 0.023)$ & $0.203( \pm 0.157)$ \\
\hline Nocardioidaceae & $1.013( \pm 0.186)$ & $2.607( \pm 0.619)$ & $1.810( \pm 0.816)$ \\
\hline Pseudomonadaceae & $0.035( \pm 0.025)$ & $0.498( \pm 0.259)$ & $0.267( \pm 0.147)$ \\
\hline Rhizobiaceae & $0.100( \pm 0.071)$ & $0.339( \pm 0.077)$ & $0.220( \pm 0.089)$ \\
\hline Rhodobacteraceae & $0.015( \pm 0.010)$ & $0.133( \pm 0.042)$ & $0.074( \pm 0.028)$ \\
\hline Trueperaceae & $5.518( \pm 4.981)$ & $0.073( \pm 0.036)$ & $2.795( \pm 2.777)$ \\
\hline Unclassified Acidimicrobidae incertae sedis & $1.690( \pm 0.252)$ & $0.571( \pm 0.093)$ & $1.131( \pm 0.892)$ \\
\hline Unclassified Actinomycetales & $\ldots$ & $0.052( \pm 0.026)$ & $0.026( \pm 0.013)$ \\
\hline Unclassified Gp 11 & $\ldots$ & $0.125( \pm 0.083)$ & $0.062( \pm 0.041)$ \\
\hline Unclassified Nocardioidaceae & $0.115( \pm 0.042)$ & $0.004( \pm 0.004)$ & $0.060( \pm 0.060)$ \\
\hline Unclassified Actinomycetales & $1.124( \pm 0.267)$ & $0.279( \pm 0.047)$ & $0.701( \pm 0.585)$ \\
\hline Unclassified Solirubacterales & $\ldots$ & $0.017( \pm 0.004)$ & $0.009( \pm 0.002)$ \\
\hline Unclassified Bacilli & $0.040( \pm 0.026)$ & $0.464( \pm 0.292)$ & $0.252( \pm 0.166)$ \\
\hline Unclassified Clostridiales & $0.025( \pm 0.025)$ & $0.060( \pm 0.024)$ & $0.043( \pm 0.024)$ \\
\hline Unclassified Gp6 & $1.259( \pm 0.551)$ & $8.967( \pm 2.200)$ & $5.113( \pm 1.729)$ \\
\hline
\end{tabular}

\footnotetext{
${ }^{a}$ Mean relative abundance based on percent of recovered sequences; SE = standard error of the mean.

${ }^{\mathrm{b}}$ Taxonomic classification level was determined based on the RDP6 taxonomy outline.

${ }^{\mathrm{c}}$ Indicates a significant difference between means of disease-conducive and -suppressive soil (CS and SS, respectively) based on Wilcoxon analysis; comparisons were statistically different at $\alpha=0.05$.
} 
samples. Nonpathogenic Actinobacteria (e.g., S. diastatochromogenes and $S$. albogriseolus) produce compounds inhibitory against $S$. scabies and contribute to disease suppression $(4,25)$. The relative abundance of Streptomyces spp. was not different between CS and SS, as shown using dilution plating (32). However, SS may have higher populations of nonpathogenic Streptomyces spp. that are associated with less severe common scab, suggesting an interaction between host plant and Streptomyces microbial communities that affects disease severity of PCS $(4,32,40)$. This population dynamic may be one of the reasons the pathogenic Streptomyces spp. were lower in SS (32). Due to the limitations of the present technology and databases, the resolution of species identity is difficult. Therefore, further studies on pathogenicity genes, community diversity, and composition targeting Streptomyces spp. may determine molecularly the relative abundance of pathogenic and nonpathogenic genera contributing to disease severity or suppression.

The phyla Firmicute, also commonly found among multiple soil samples in other work $(20,57)$, include bacilli that were among the most abundant in both CS and SS samples. One genus of Bacillus was significantly higher in CS by an order of magnitude, whereas a yet-to-be-classified bacillus was found to have a higher relative abundance in SS. Previous studies found that metabolites (iturin A) produced by Bacillus sp. sunhua showed antifungal activity against Fusarium oxysporum, the causal organism of dry rot disease in potato; S. scabies; and other pathogens of potato (13). Bacillus sp. sunhua is beneficial for potato disease control under field conditions (13). The results presented here indicate that this specific site, at the level of genus bacilli in the soil, may not necessarily reflect the individual species of functional importance to endemic soil disease suppression.

This study reveals variation in bacterial communities associated with potato fields of CS and SS. Characterizing microbial composition and diversity, spatial patterns, and the distribution of microbial diversity $(9,48)$ in disease-suppressive soil is an important first step to understanding the potential impacts of these communities on plant fitness. This will help to develop disease management practices to reduce pathogen populations in soil and, therefore, reduce the economic and environmental impacts of plant disease control.

\section{Acknowledgments}

This project was partially supported by the Michigan Potato Industry Commission and the Michigan State University Project GREEEN. Additional

Table 5. Relative sequence abundance of significantly different bacteria from soil samples divided into categories of soil disease profile and combined totals

\begin{tabular}{|c|c|c|c|}
\hline \multirow[b]{2}{*}{ Genus $^{\mathrm{b}}$} & \multicolumn{3}{|c|}{ Mean relative abundance $(\mathrm{SE})^{\mathrm{a}}$} \\
\hline & $\mathbf{C S}^{\mathbf{c}}$ & $\mathbf{S S}^{\mathbf{c}}$ & Total \\
\hline Actinospica & $0.206( \pm 0.096)$ & $0.009( \pm 0.005)$ & $0.107( \pm 0.105)$ \\
\hline Aeromicrobium & $0.030( \pm 0.012)$ & $0.193( \pm 0.065)$ & $0.112( \pm 0.047)$ \\
\hline Agromyces & & $0.021( \pm 0.007)$ & $0.011( \pm 0.003)$ \\
\hline Amaricoccus & $0.005( \pm 0.005)$ & $0.094( \pm 0.038)$ & $0.050( \pm 0.022)$ \\
\hline Aminobacter & $0.005( \pm 0.005)$ & $\ldots$ & $0.003( \pm 0.003)$ \\
\hline Bacillus & $1.374( \pm 0.814)$ & $0.112( \pm 0.013)$ & $0.743( \pm 0.693)$ \\
\hline Cohnella & $0.070( \pm 0.023)$ & $0.009( \pm 0.005)$ & $0.039( \pm 0.038)$ \\
\hline Deinococcus & $0.110( \pm 0.055)$ & $0.009( \pm 0.005)$ & $0.059( \pm 0.058)$ \\
\hline Lysobacter & $0.040( \pm 0.029)$ & $0.206( \pm 0.053)$ & $0.123( \pm 0.046)$ \\
\hline Microvirga & $0.035( \pm 0.015)$ & $0.240( \pm 0.083)$ & $0.138( \pm 0.059)$ \\
\hline Mycobacterium & $0.296( \pm 0.049)$ & $0.116( \pm 0.023)$ & $0.206( \pm 0.160)$ \\
\hline Nitrobacter & $0.135( \pm 0.047)$ & $0.017( \pm 0.004)$ & $0.076( \pm 0.070)$ \\
\hline Novosphingobium & & $0.017( \pm 0.004)$ & $0.009( \pm 0.002)$ \\
\hline Pedomicrobium & $0.020( \pm 0.009)$ & $0.236( \pm 0.085)$ & $0.128( \pm 0.052)$ \\
\hline Phyllobacterium & & $0.064( \pm 0.043)$ & $0.032( \pm 0.022)$ \\
\hline Rhizobium & $0.075( \pm 0.069)$ & $0.240( \pm 0.054)$ & $0.158( \pm 0.065)$ \\
\hline Sporacetigenium & $0.080( \pm 0.022)$ & $0.009( \pm 0.005)$ & $0.044( \pm 0.043)$ \\
\hline Symbiobacterium & $0.030( \pm 0.018)$ & $0.150( \pm 0.030)$ & $0.090( \pm 0.030)$ \\
\hline Truepera & $5.518( \pm 4.981)$ & $0.073( \pm 0.036)$ & $2.795( \pm 2.777)$ \\
\hline Unclassified Sorangiineae & $0.005( \pm 0.005)$ & $0.133( \pm 0.056)$ & $0.069( \pm 0.031)$ \\
\hline Unclassified 112 & $0.145( \pm 0.072)$ & $0.764( \pm 0.281)$ & $0.455( \pm 0.213)$ \\
\hline Unclassified WS3 & $0.040( \pm 0.026)$ & $0.447( \pm 0.289)$ & $0.243( \pm 0.165)$ \\
\hline Unclassified $\beta$-Proteobacteria & $0.010( \pm 0.010)$ & $0.060( \pm 0.021)$ & $0.035( \pm 0.015)$ \\
\hline Unclassified Ilumatobacter & $\ldots$ & $0.086( \pm 0.018)$ & $0.043( \pm 0.009$ \\
\hline Unclassified Gp 11 & $\ldots$ & $0.125( \pm 0.083)$ & $0.062( \pm 0.041)$ \\
\hline Unclassified Nocardioides & $0.231( \pm 0.050)$ & $0.752( \pm 0.141)$ & $0.491( \pm 0.186)$ \\
\hline Unclassified Solirubacterales & $1.701( \pm 0.268)$ & $0.571( \pm 0.088)$ & $1.136( \pm 0.894)$ \\
\hline Unclassified BRC1 & & $0.052( \pm 0.026)$ & $0.026( \pm 0.013)$ \\
\hline Unclassified Chloroflexi & $0.005( \pm 0.005)$ & $0.064( \pm 0.018)$ & $0.035( \pm 0.011)$ \\
\hline Unclassified Thermomicrobia & $0.712( \pm 0.222)$ & $0.150( \pm 0.037)$ & $0.431( \pm 0.375)$ \\
\hline Unclassified 45 & $0.281( \pm 0.038)$ & $0.082( \pm 0.022)$ & $0.181( \pm 0.151)$ \\
\hline Unclassified Planococcaceae & $0.090( \pm 0.028)$ & $0.013( \pm 0.005)$ & $0.052( \pm 0.048)$ \\
\hline Unclassified Bacillaceae & $1.349( \pm 0.262)$ & $0.305( \pm 0.042)$ & $0.827( \pm 0.695)$ \\
\hline Unclassified Clostridiaceae & $0.065( \pm 0.017)$ & $0.004( \pm 0.004)$ & $0.035( \pm 0.035)$ \\
\hline Unclassified Gp 4 & $0.226( \pm 0.114)$ & $2.328( \pm 0.567)$ & $1.277( \pm 0.396)$ \\
\hline Unclassified Bradyrhizobiaceae & $2.624( \pm 0.331)$ & $0.515( \pm 0.186)$ & $1.569( \pm 1.405)$ \\
\hline Unclassified Rhodobacteraceae & & $0.030( \pm 0.011)$ & $0.015( \pm 0.005)$ \\
\hline Unclassified Acetobacteraceae & $0.742( \pm 0.062)$ & $0.344( \pm 0.045)$ & $0.543( \pm 0.393)$ \\
\hline Unclassified Gp 6 & $1.259( \pm 0.551)$ & $8.967( \pm 2.200)$ & $5.113( \pm 1.729)$ \\
\hline Unclassified Sphingomonadaceae & $0.005( \pm 0.005)$ & $0.039( \pm 0.013)$ & $0.022( \pm 0.009)$ \\
\hline Unclassified Comamonadaceae & $0.005( \pm 0.005)$ & $0.103( \pm 0.036)$ & $0.054( \pm 0.021)$ \\
\hline Unclassified Acetobacteraceae & $0.497( \pm 0.123)$ & $0.060( \pm 0.014)$ & $0.278( \pm 0.255)$ \\
\hline
\end{tabular}

${ }^{a}$ Mean relative abundance based on percentage of recovered sequences; SE = standard error of the mean.

b Taxonomic classification level was determined based on the RDP6 taxonomy outline; unclassified genera identities were based on the highest taxonomic level of resolution.

${ }^{c}$ Indicates a significant difference between means of disease-conducive and -suppressive soil (CS and SS, respectively) based on Wilcoxon analysis; comparisons were statistically different at $\alpha=0.05$. 
resources and computational analyses were provided through the University of Minnesota Supercomputing Institute, Michigan State University's Center for Microbial Ecology, and Michigan State University's Ribosomal Database Project. We thank P. Schloss, University of Michigan, for discussions on data analysis; J. D. Munoz, Michigan State University, for statistical consulting; and M. Chilvers, Michigan State University, and Z. K. Atallah, Hartnell College, for critical review of this article.

\section{Literature Cited}

1. Alabouvette, C., and Hornby, D. 1990. Pages 27-43 in: Biological Control of Fusarium Wilt Pathogens in Suppressive Soils. CAB International, Oxfordshire, UK

2. Atkinson, G. F. 1892. Some Diseases of Cotton. Agricultural Experiment Station of the Alabama Agricultural and Mechanical College.

3. Boudreau, M., and Andrews, J. 1987. Factors influencing antagonism of Chaetomium globosum to Venturia inaequalis: A case study in failed biocontrol. Phytopathology 77:1470-1475.

4. Bowers, J., Kinkel, L., and Jones, R. 1996. Influence of disease-suppressive strains of Streptomyces on the native Streptomyces community in soil as determined by the analysis of cellular fatty acids. Can. J. Microbiol. 42:27-37.

5. Buée, M., Reich, M., Murat, C., Morin, E., Nilsson, R. H., Uroz, S., and Martin, F. 2009. 454 Pyrosequencing analyses of forest soils reveal an unexpectedly high fungal diversity. New Phytol. 184:449-456.

6. Chao, A. 1984. Nonparametric estimation of the number of classes in a population. Scand. J. Stat. 11:265-270.

7. Chao, A., and Lee, S. M. 1992. Estimating the number of classes via sample coverage. J. Am. Stat. Assoc. 87:210-217.

8. Cook, R., and Rovira, A. 1976. The role of bacteria in the biological control of Gaeumannomyces graminis by suppressive soils. Soil Biol. Biochem. $8: 269-273$.

9. Ettema, C. H., and Wardle, D. A. 2002. Spatial soil ecology. Trends Ecol. Evol. 17:177-183.

10. Faoro, H., Alves, A., Souza, E., Rigo, L., Cruz, L., Al-Janabi, S., Monteiro, R., Baura, V., and Pedrosa, F. 2010. Influence of soil characteristics on the diversity of bacteria in the Southern Brazilian Atlantic For. Appl. Environ. Microbiol. 76:4744-4749.

11. Gans, J., Wolinsky, M., and Dunbar, J. 2005. Computational improvements reveal great bacterial diversity and high metal toxicity in soil. Science 309:1387-1390.

12. Grayston, S. J., Wang, S., Campbell, C. D., and Edwards, A. C. 1998. Selective influence of plant species on microbial diversity in the rhizosphere. Soil Biol. Biochem. 30:369-378.

13. Han, J., Cheng, J., Yoon, T., Song, J., Rajkarnikar, A., Kim, W., Yoo, I., Yang, Y., and Suh, J. 2005. Biological control agent of common scab disease by antagonistic strain Bacillus sp. sunhua. J. Appl. Microbiol. 99:213221

14. Hiltunen, L., Ojanperä, T., Kortemaa, H., Richter, E., Lehtonen, M., and Valkonen, J. 2009. Interactions and biocontrol of pathogenic Streptomyces strains co occurring in potato scab lesions. J. Appl. Microbiol. 106:199-212.

15. Hornby, D. 1983. Suppressive soils. Annu. Rev. Phytopathol. 21:65-85.

16. Huse, S., Dethlefsen, L., Huber, J., Welch, D., Relman, D., and Sogin, M. 2008. Exploring microbial diversity and taxonomy using SSU rRNA hypervariable tag sequencing. PLoS Genet. 4:e1000255. doi:10.1371/journal. pgen. 1000255

17. Jones, R. T., Robeson, M. S., Lauber, C. L., Hamady, M., Knight, R., and Fierer, N. 2009. A comprehensive survey of soil acidobacterial diversity using pyrosequencing and clone library analyses. ISME J. 3:442-453.

18. Kloepper, J., Rodriguez-Kabana, R., Zehnder, A., Murphy, J., Sikora, E., and Fernandez, C. 1999. Plant root-bacterial interactions in biological control of soilborne diseases and potential extension to systemic and foliar diseases. Aust. Plant Pathol. 28:21-26.

19. Kuske, C. R., Ticknor, L. O., Miller, M. E., Dunbar, J. M., Davis, J. A., Burns, S. M., and Belnap, J. 2002. Comparison of soil bacterial communities in rhizospheres of three plant species and the interspaces in an arid grassland. Appl. Environ. Microbiol. 68:1854-1863.

20. Lauber, C. L., Hamady, M., Knight, R., and Fierer, N. 2009. P Pyrosequencing-based assessment of soil $\mathrm{pH}$ as a predictor of soil bacterial community structure at the continental scale. Appl. Environ. Microbiol. 75:5111-5120.

21. Lee, S. H., Ka, J. O., and Cho, J. C. 2008. Members of the phylum Acidobacteria are dominant and metabolically active in rhizosphere soil. FEMS Microbiol. Lett. 285:263-269.

22. Liu, D., Anderson, N., and Kinkel, L. 1995. Biological control of potato scab in the field with antagonistic Streptomyces scabies. Phytopathology 85:827-831.

23. Liu, Z. Z., DeSantis, T. Z., Andersen, G. L., and Knight, R. 2008. Accurate taxonomy assignments from $16 \mathrm{~S}$ rRNA sequences produced by highly parallel pyrosequencers. Nucleic Acids Res. 36:e120.

24. Long, H. H., Sonntag, D. G., Schmidt, D. D., and Baldwin, I. T. 2010. The structure of the culturable root bacterial endophyte community of Nicotiana attenuata is organized by soil composition and host plant ethylene production and perception. New Phytol. 185:554-567.

25. Lorang, J., Anderson, N., Lauer, F., and Wildung, D. 1989. Disease decline in a Minnesota potato scab plot. Am. Potato J. 66:531.
26. Loria, R., Bukhalid, R., Fry, B., and King, R. 1997. Plant pathogenicity in the genus Streptomyces. Plant Dis. 81:836-846.

27. Manter, D. K., Delgado, J. A., Holm, D. G., and Strong, R. A. 2010. Pyrosequencing reveals a highly diverse and cultivar-specific bacterial endophyte community in potato roots. Microb. Ecol. 60:157-166.

28. Marschner, P., Yang, C. H., Lieberei, R., and Crowley, D. E. 2001. Soil and plant specific effects on bacterial community composition in the rhizosphere. Soil Biol. Biochem. 33:1437-1445.

29. Mazzola, M. 2002. Mechanisms of natural soil suppressiveness to soilborne diseases. Antonie Leeuwenhoek 81:557-564.

30. Mazzola, M., Cook, R., Thomashow, L., Weller, D., and Pierson, L., 3rd. 1992. Contribution of phenazine antibiotic biosynthesis to the ecologica competence of fluorescent pseudomonads in soil habitats. Appl. Environ. Microbiol. 58:2616-2624.

31. Mendes, R., Kruijt, M., de Bruijn, I., Dekkers, E., van der Voort, M., Schneider, J. H. M., Piceno, Y. M., DeSantis, T. Z., Andersen, G. L., and Bakker, P. A. H. M. 2011. Deciphering the rhizosphere microbiome for disease-suppressive bacteria. Science 332:1097-1100.

32. Meng, Q. M., Yin, J. F., Rosenzweig, N., Douches, D., and Hao, J. J. 2012. Culture-based assessment of microbial communities in soil suppressive to potato common scab. Plant Dis. 96:712-717.

33. Menzies, J. 1959. Occurrence and transfer of a biological factor in soil that suppresses potato scab. Phytopathology 49:648-652.

34. Postma, J., Schilder, M. T., Bloem, J., and van Leeuwen-Haagsma, W. K. 2008. Soil suppressiveness and functional diversity of the soil microflora in organic farming systems. Soil Biol. Biochem. 40:2394-2406.

35. Raaijmakers, J. M., and Weller, D. M. 1998. Natural plant protection by 2, 4-diacetylphloroglucinol-producing Pseudomonas spp. in take-all decline soils. Mol. Plant-Microbe Interact. 11:144-152.

36. Sanguin, H., Sarniguet, A., Gazengel, K., Moënne Loccoz, Y., and Grundmann, G. 2009. Rhizosphere bacterial communities associated with disease suppressiveness stages of take all decline in wheat monoculture. New Phytol. 184:694-707.

37. Sarniguet, A., and Lucas, P. 1992. Evaluation of populations of fluorescent pseudomonads related to decline of take-all patch on turfgrass. Plant Soil 145:11-15.

38. Schlatter, D., Fubuh, A., Xiao, K., Hernandez, D., Hobbie, S., and Kinkel, L. 2009. Resource amendments influence density and competitive phenotypes of Streptomyces in soil. Microb. Ecol. 57:413-420.

39. Schloss, P. D., Westcott, S. L., Ryabin, T., Hall, J. R., Hartmann, M., Hollister, E. B., Lesniewski, R. A., Oakley, B. B., Parks, D. H., Robinson, C. J. Sahl, J. W., Stres, B., Thallinger, G. G., Van Horn, D. J., and Weber, C. F. 2009. Introducing mothur: open source, platform-independent, communitysupported software for describing and comparing microbial communities Appl. Environ. Microbiol. 75:7537-7754.

40. Schottel, J. L., Shimizu, K., and Kinkel, L. L. 2001. Relationships of in vitro pathogen inhibition and soil colonization to potato scab biocontrol by antagonistic Streptomyces spp. Biol. Control 20:102-112.

41. Shannon, C. E. 1948. A mathematical theory of communication. Bell Syst. Tech. J. 27:379-423, 623-656.

42. Simpson, E. H. 1949. Measurement of diversity. Nature 163:688.

43. Slack, S. 1992. A look at potato leafroll virus and PVY: past, present, and future. Valley Potato Grower 57:35-39.

44. Stevenson, W., Loria, R., Franc, G., and Weingartner, D. 2001. Compendium of Potato Diseases. American Phytopathological Society, St. Paul $\mathrm{MN}$.

45. Sugiyama, A., Vivanco, J. M., Jayanty, S. S., and Manter, D. K. 2010. Pyrosequencing assessment of soil microbial communities in organic and conventional potato farms. Plant Dis. 94 :1329-1335.

46. Tagawa, M., Tamaki, H., Manome, A., Koyama, O., and Kamagata, Y. 2010. Isolation and characterization of antagonistic fungi against potato scab pathogens from potato field soils. FEMS Microbiol. Lett. 305:136-142.

47. Tiedje, J. M., Asuming-Brempong, S., Nusslein, K., Marsh, T. L., and Flynn, S. J. 1999. Opening the black box of soil microbial diversity. Appl Soil Ecol. 13:109-122.

48. Torsvik, V., and Ovreas, L. 2002. Microbial diversity and function in soil from genes to ecosystems. Curr. Opin. Microbiol. 5:240-245.

49. Van Bruggen, A., and Semenov, A. 2000. In search of biological indicators for soil health and disease suppression. Appl. Environ. Microbiol. 15:13-24.

50. Walker, J., and Snyder, W. 1934. Pea wilt much less severe on certain soils. Univ. Wisc. Agric. Exp. Stn. Bull. 428

51. Wang, Q., Garrity, G. M., Tiedje, J. M., and Cole, J. R. 2007. Naive Bayesian classifier for rapid assignment of rRNA sequences into the new bacterial taxonomy. Appl. Environ. Microbiol. 73:5261-5267.

52. Wanner, L. 2006. A survey of genetic variation in Streptomyces isolates causing potato common scab in the United States. Phytopathology 96:13631371

53. Wanner, L. 2007. High proportions of nonpathogenic Streptomyces are associated with common scab-resistant potato lines and less severe disease. Can. J. Microbiol. 53:1062-1075.

54. Wanner, L. A., and Haynes, K. G. 2009. Aggressiveness of Streptomyces on four potato cultivars and implications for common scab resistance breeding. Am. J. Pot. Res. 86:335-346.

55. Weller, D., Raaijmakers, J., Gardener, B., and Thomashow, L. 2002. Micro- 
bial populations responsible for specific soil suppressiveness to plant pathogens 1. Annu. Rev. Phytopathol. 40:309-348.

56. Weller, D. M., Landa, B., Mavrodi, O., Schroeder, K., De La Fuente, L., Blouin Bankhead, S., Allende Molar, R., Bonsall, R., Mavrodi, D., and Thomashow, L. 2007. Role of 2,4 diacetylphloroglucinol producing fluorescent Pseudomonas spp. in the defense of plant roots. Plant Biol. 9:4-20.

57. Yin, C., Jones, K. L., Peterson, D. E., Garrett, K. A., Hulbert, S. H., and Paulitz, T. C. 2010. Members of soil bacterial communities sensitive to till- age and crop rotation. Soil Biol. Biochem. 42:2111-2118.

58. Zhang, L., and Xu, Z. 2008. Assessing bacterial diversity in soil: assessing bacterial diversity in soil: a brief review (Soils, Sec. 1 "Soil organic matter dynamics"). J. Soils Sedim. 8:379-388.

59. Zhang, Q., Yang, R. Y., Tang, J. J., Yang, H. S., Hu, S. J., and Chen, X. 2010. Positive feedback between mycorrhizal fungi and plants influences plant invasion success and resistance to invasion. PLoS ONE 5 e12380. doi:10.1371/journal.pone.0012380. 